\title{
Positional preferences of acetyl esterases from different CE families towards acetylated 4-O-methyl glucuronic acid-substituted xylo-oligosaccharides
}

\author{
Klaus G Neumüller ${ }^{1,2}$, Adriana Carvalho de Souza', Jozef HJ van Rijn ${ }^{1}$, Hugo Streekstra ${ }^{1}$, Harry Gruppen² \\ and Henk A Schols ${ }^{2 *}$
}

\begin{abstract}
Background: Acetylation of the xylan backbone restricts the hydrolysis of plant poly- and oligosaccharides by hemicellulolytic enzyme preparations to constituent monosaccharides. The positional preferences and deacetylation efficiencies of acetyl esterases from seven different carbohydrate esterase (CE) families towards different acetylated xylopyranosyl units (Xylp) - as present in 4-O-methyl-glucuronic acid (MeGlcA)-substituted xylo-oligosaccharides (AcUXOS) derived from Eucalyptus globulus - were monitored by ${ }^{1} \mathrm{H}$ NMR, using common conditions for biofuel production ( $\left.\mathrm{pH} 5.0,50^{\circ} \mathrm{C}\right)$.

Results: Differences were observed regarding the hydrolysis of 2-O, 3-O, and 2,3-di-O acetylated Xylp and 3-O acetylated Xylp 2-O substituted with MeGlcA. The acetyl esterases tested could be categorized in three groups having activities towards (i) 2-O and 3-O acetylated Xylp, (ii) 2-O, 3-O, and 2,3-di-O acetylated Xylp, and (iii) 2-O, 3-O, and 2,3-di-O acetylated Xylp, as well as 3-O acetylated Xylp 2-O substituted with MeGlcA at the non-reducing end. A high deacetylation efficiency of up to $83 \%$ was observed for CE5 and CE1 acetyl esterases. Positional preferences were observed towards 2,3-di-O acetylated Xylp (TeCE1, AnCE5, and OsCE6) or 3-O acetylated Xylp (CtCE4).

Conclusions: Different positional preferences, deacetylation efficiencies, and initial deacetylation rates towards 2-O, 3-O, and 2,3-di-O acetylated Xylp and 3-O acetylated Xylp 2-O substituted with MeGlcA were demonstrated for acetyl esterases from different $\mathrm{CE}$ families at $\mathrm{pH} 5.0$ and $50^{\circ} \mathrm{C}$. The data allow the design of optimal, deacetylating hemicellulolytic enzyme mixtures for the hydrolysis of non-alkaline-pretreated bioenergy feedstocks.
\end{abstract}

Keywords: Acetyl xylan esterase, Positional specificity, Xylan, Deacetylation, Hemicellulase

\section{Background}

Xylan is a valuable source of C5 sugars for use in biorefineries. However, acetylation of xylan restricts enzymatic degradation of the xylan backbone [1]. Therefore, enzymatic hydrolysis of glycosidic substituents and phenyl and acetyl esters increases the accessibility of the (1 through 4)- $\beta$-D-xylan backbone for $\beta$-1,4-endo-xylanases and $\beta-1,4$-xylosidases. The Carbohydrate-Active enzymes (CAZy) database classification (www.cazy.org) recognizes 16 carbohydrate esterase (CE) families. Unfortunately, a lack of knowledge of the sequence-to-specificity

\footnotetext{
*Correspondence: Henk.Schols@wur.nl

¿2aboratory of Food Chemistry, Wageningen University, Bornse Weilanden 9, 6708 WG Wageningen, The Netherlands

Full list of author information is available at the end of the article
}

relationships in CAZy families does not yet allow a reliable, automated substrate prediction [2] for the esterases within a CE family.

Carbohydrate esterases with activity towards acetylated xylan were reported to fall into eight different CE families (CE1, 2, 3, 4, 5, 6, 7, and 16) [3,4]. As reviewed previously [3], the positional preference on synthetic substrates has been described for some acetyl xylan esterases. A characterization of the positional preference of acetyl xylan esterases on partially acetylated xylooligosaccharides by NMR is only available for the CE1 (Schizophyllum commune), CE4 (Streptomyces lividans), and CE6 (Orpinomyces sp.) esterases [5,6]. In only a few cases has the specificity towards different acetylated xylopyranosyl units $(\mathrm{Xyl} p)$ also been investigated on 
actual plant oligosaccharides $[7,8]$, and even in those cases the deacetylation rates towards the differently acetylated Xyl $p$ were not reported. Knowledge of these rates is required to determine the positional preference of the acetyl esterases in the early stage of the reaction when many competing substrates are still present.

Using artificial substrates, it has been shown that CE1 and CE5 acetyl xylan esterases have a strong preference for the deacetylation of 2-O acetyl 4-nitrophenyl $\beta$-Dxylopyranoside [9]. However, esterases from these families were reported to also be active on 3-O acetylated xylopyranosyl residues if plant-derived xylo-oligosaccharides were used [7]. This shows that one cannot rely solely on artificial substrates to characterize the positional preference of acetyl esterases, and that natural substrates are required as well. Both the initial deacetylation rates and the final deacetylation efficiency on differently acetylated, plant-derived Xyl $p$ residues are required to characterize the acetyl esterases from different $\mathrm{CE}$ families. Moreover, these measurements should preferably be performed under hydrolysis conditions relevant for the production of industrial biofuels, since it cannot be excluded that these conditions affect the positional preference.

Recently, it was shown that supplementation of a hemicellulolytic enzyme preparation containing CE1 acetyl xylan esterases with a CE5 acetyl xylan esterase from Trichoderma longibrachiatum increased substrate conversion to monomers [10]. Addition of acetyl xylan esterases to hemicellulolytic enzyme preparations allows efficient hydrolysis of hemicellulose when harsh alkaline pretreatments [11] are reduced. This shows the potential relevance of (the right mix of) acetyl esterases for efficient hemicellulose degradation.

Here, we describe the deacetylation by carbohydrate esterases from different $\mathrm{CE}$ families on acetylated 4-O-methyl glucuronic acid (MeGlcA)-substituted xylooligosaccharides (AcUXOS) at $\mathrm{pH} 5$ and $50^{\circ} \mathrm{C}$, which are common conditions for biofuel production $[12,13]$ and conditions under which the acetyl esterases are active. ${ }^{1} \mathrm{H}$ and 2D NMR spectra were used to characterize the deacetylation of 2-O, 3-O, and 2,3-di-O acetylated Xyl $p$ and the 3-O acetylated Xyl $p 2-O$ substituted with MeGlcA. The positional preference by the enzymes towards differently acetylated $\mathrm{Xyl} p$ is presented.

\section{Results and discussion}

\section{Acetyl esterases}

Selected acetyl esterases were analyzed for their purity by SDS-PAGE (Figure 1). The protein bands of $34 \mathrm{kDa}$, $34 \mathrm{kDa}, 26 \mathrm{kDa}$, and $32 \mathrm{kDa}$ matched with the molecular mass reported previously for $\operatorname{TrCE} 5(34 \mathrm{kDa})$ [14] and with the calculated molecular masses based on the protein sequences, which were $33.8 \mathrm{kDa}(A n \mathrm{CE} 16)$, $25.8 \mathrm{kDa}(\mathrm{AnCE} 5)$, and $32.5 \mathrm{kDa}(\mathrm{TeCE} 1)$. For the commercially available acetyl esterases, protein bands were

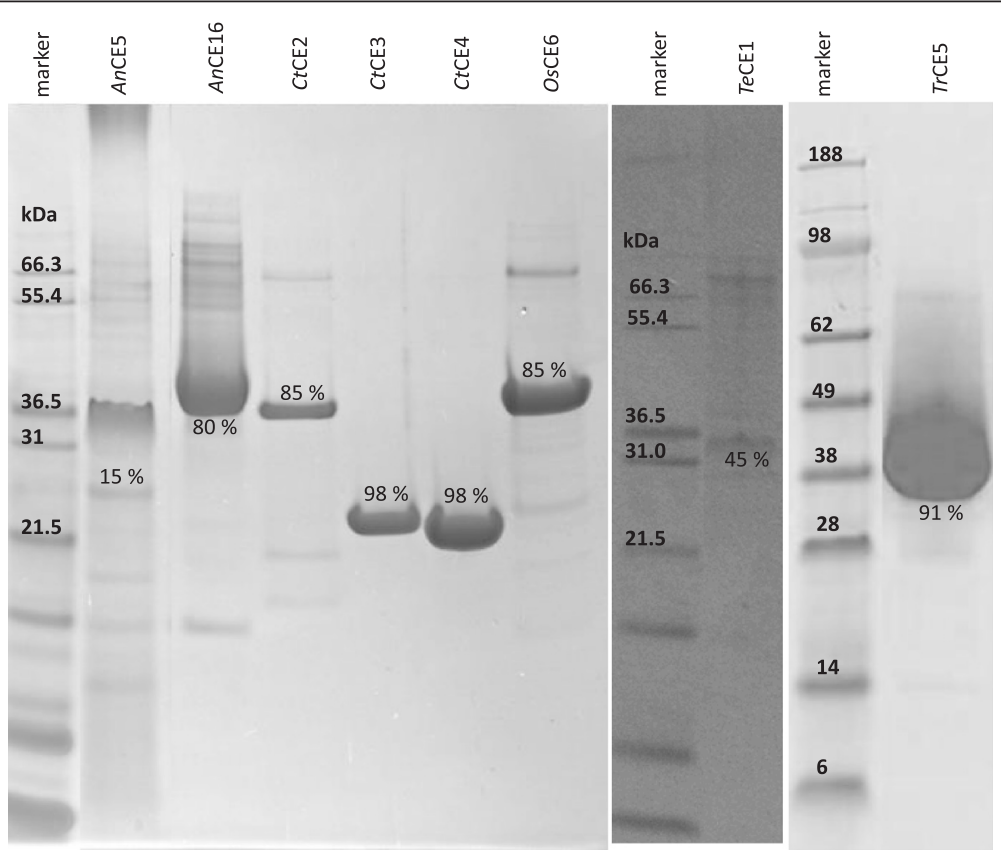

Figure 1 SDS-PAGE acetyl esterases. SDS-PAGE of acetyl esterases from Aspergillus niger (AnCE5, AnCE16), Clostridium thermocellum (CtCE2, CtCE3, CtCE4), Orpinomyces sp. (OsCE6), Talaromyces emersonii (TeCE1), and Trichoderma reesei (TrCE5). The purity of the acetyl esterases present is shown as densimetric proportion (\%) of the total proteins present as determined by SDS-PAGE analysis. The protein markers Mark 12 and SeeBlue Plus 2 were used. 
observed at $37 \mathrm{kDa}, 24 \mathrm{kDa}, 24 \mathrm{kDa}$, and $34 \mathrm{kDa}$, respectively, and these also matched with the calculated molecular masses based on the protein sequences, which were $36.4 \mathrm{kDa}$ (CtCE2), $22.8 \mathrm{kDa}$ (CtCE3), $22.8 \mathrm{kDa}$ (CtCE4), and $34.9 \mathrm{kDa}$ (OsCE6).

The purity of the acetyl esterases present in the enzyme solutions was determined by quantitative densitometry, shown in Figure 1 as the proportion (\%) of the total proteins present. The SDS-PAGE analysis of the cloned acetyl esterases expressed by A. niger ISO527 also showed the presence of protein bands different from those of the cloned enzymes (Figure 1). Particularly for $A n C E 5$, a range of other protein bands was present, resulting in a lower purity (15\%) compared to the other acetyl esterase preparations. Nevertheless, the production of the recombinant enzymes is believed to be functionally specific, since production of native enzymes acting on complex carbohydrates is not induced by the expression and cultivation system used (no further data shown) [15]. An assay to verify the absence of other monosaccharide-releasing side activities was performed. No monosaccharide-releasing activities were apparent after incubation of water unextractable solids (WUS) with the acetyl esterase-containing solutions ( $\operatorname{Tr} \mathrm{CE} 1$, CtCE2, CtCE3, CtCE4, TrCE5, AnCE5, OsCE6, and $A n C E 16$; results not shown). This confirms a selective secretion of the cloned esterases by A. niger ISO527, as observed previously [15]. No other acetyl esterases are assumed to be present due to the specific protein expression and the absence of deviating or unexpected effects during long (16-h) incubation times, monitored by NMR analysis, as described below. The absence of interfering side activities and the presence of significant amounts of each acetyl esterase enabled investigation of their modes of action.

\section{Activity of acetyl esterases Activity on p-nitrophenyl acetate ( $p$-NP-Ac)}

The generic esterase substrate $p$-NP-Ac was used to verify the presence of acetyl esterase activity. The release of $p$-nitrophenol by the hydrolysis of $p$-NP-Ac is shown in Figure 2. The serine-type acetyl esterases, $\mathrm{TeCE} 1, \mathrm{CtCE} 2$, $C t \mathrm{CE} 3, A n \mathrm{CE} 5, \operatorname{TrCE} 5$, OsCE6, and $A n \mathrm{CE} 16$, readily hydrolyzed $p$-NP-Ac. The non-serine-type, metal-dependent esterase $C t C E 4$ was not active on $p$-NP-Ac. The highest $p$-nitrophenol release was observed for the bacterial CE2 and CE3 esterases from $C$. thermocellum (CtCE2 and $C t \mathrm{CE} 3$ ). The CE5 acetyl xylan esterases (AnCE5 and $\operatorname{TrCE5}$ ) hydrolyzed $p$-NP-Ac more efficiently than TeCE1, OsCE6, and $A n C E 16$. The hydrolysis of $p$-NP-Ac only gives a rough indication of the expected hydrolytic activity on acetylated oligosaccharides. Hence, characterization of the acetyl esterases was continued with partially acetylated plant xylo-oligosaccharides.

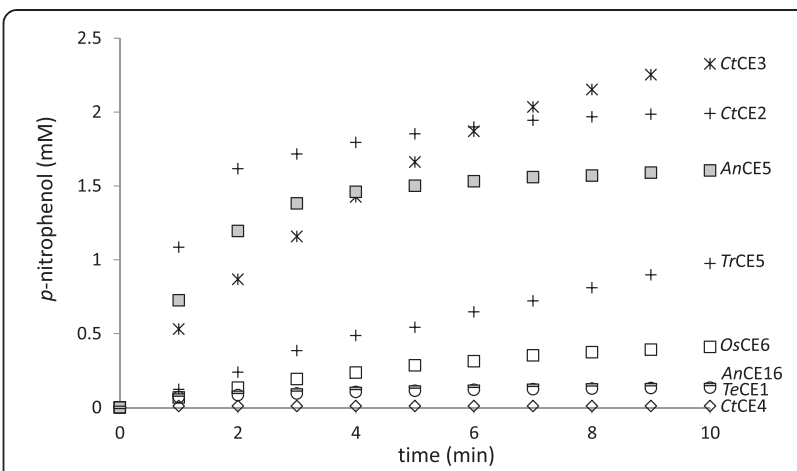

Figure $2 \boldsymbol{p}$-Nitrophenyl acetate assay. $p$-Nitrophenol released by incubation of $p$-nitrophenyl acetate with acetyl esterases (TeCE1, CtCE2, CtCE3, CtCE4, TrCE5, AnCE5, OsCE6, and AnCE16).

\section{Deacetylation efficiency of acetylated xylo-oligosaccharides}

The acetic acid released from AcUXOS after an incubation time of $16 \mathrm{~h}$ with acetyl esterases from different families as determined by ${ }^{1} \mathrm{H}$ NMR is shown in Figure 3. All acetyl esterases showed deacetylation of AcUXOS. High levels of acetic acid release were obtained with the fungal $T e C E 1, T r C E 5$, and $A n C E 5$ and the bacterial $C t C E 4$. The efficient deacetylation by CE5 esterases is in agreement with the reported high deacetylation of polyand oligosaccharides by Axe1 from T. longibrachiatum, which belongs to CE family 5 [10]. CtCE4 efficiently deacetylated AcUXOS, in contrast to its absence of activity towards $p$-NP-Ac (Figure 2). This indicates that activities by acetyl esterases can differ significantly when they act on acetylated oligosaccharides or on generic substrates.

Moderate acetic acid release was obtained for CtCE2, OsCE6, and AnCE16 (Figure 3). Hydrolytic activity by CtCE2 from Neocallimastix patriciarum towards acetylated glucuronoxylan has been reported before [16]. OsCE6 has been reported to remove acetyl groups from 2-O, 3-O, and 2,3-di- $O$ acetylated Xyl $p[5,6]$.

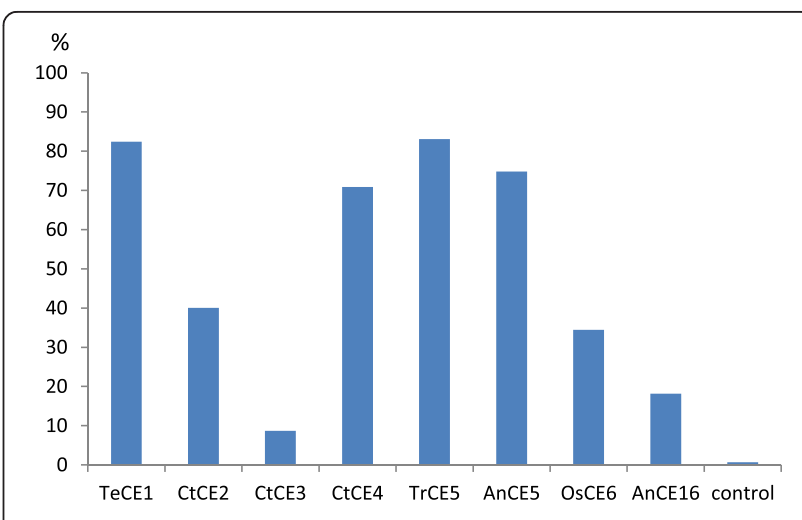

Figure 3 Acetic acid assay. Acetic acid released as proportion (\%) of the total amount of acetic acid present in acetylated 4-O-methyl glucuronic acid (MeGlcA)-substituted xylo-oligosaccharide (AcUXOS) after an incubation time of $16 \mathrm{~h}$, at $\mathrm{pH} 5.0$ and $50^{\circ} \mathrm{C}$. 
Although the positions of all acetyl groups over the oligomeric substrate AcUXOS are not known, the number of acetyl groups removed by $A n C E 16$ (18\%) matches with the level of reducing end groups present as estimated from the mass spectrum obtained previously [8]. The data suggest exo-acting activity for the CE16 enzyme from Aspergillus niger, as proposed previously for the CE16 acetyl esterase from Trichoderma reesei [17]. So far, there is no structural information available for the CE16 family [18], indicating a further need for characterization of this CE family. Only low deacetylation was obtained for $C t C E 3$, showing that this enzyme is not efficiently de-esterifying AcUXOS under the hydrolysis conditions applied.

\section{Structural characterization of AcUXOS}

The AcUXOS fraction was further used to determine the positional specificity of acetyl esterases. In order to characterize the acetylation pattern of AcUXOS, ${ }^{1} \mathrm{H}$ and 2D NMR measurements were performed. The relevant acetylated structural units of the eucalyptus xylooligosaccharides were assigned using literature data [19] and confirmed by the reporter peaks observed for the AcUXOS fraction by ${ }^{1} \mathrm{H}$ and ${ }^{13} \mathrm{C}$ heteronuclear multiplebond correlation spectroscopy (HMBC) spectra (Table 1, Figure 4). Due to the focus of this study on monitoring the deacetylation of the structural units by various enzymes in time, a full assignment of all cross peaks was not needed. The presence of the targeted structural units was confirmed by reporter peaks (Table 1), which were in agreement with literature data $[5,19]$. The acetyl positions and their corresponding chemical shifts for the observed 2-O, 3-O, and 2,3-di-O acetylated Xyl $p$ and the 3-O acetylated $\alpha-1,2-\mathrm{MeGl}$ A-substituted Xyl $p$ residues are shown in Table 1 . The regions used for the integration of the different acetylated structural units $\left({ }^{1} \mathrm{H}\right.$ NMR and ${ }^{13} \mathrm{C}$ HMBC spectra) are shown in Figure 4. The NMR measurement showed that approximately 26\% of the acetyl groups present in AcUXOS are attached at $O-2$ and $46 \%$ at $O-3$ of mono-acetylated Xyl $p, 15 \%$ represent di-acetylated $\mathrm{Xyl} p$, and $13 \%$ are attached at $O-3$ of $\alpha-1,2-M e G l c A-s u b s t i t u t e d \mathrm{Xyl} p$. Discrimination between acetyl groups present on "internal" Xyl $p$ or on $\mathrm{Xyl} p$ at the reducing and non-reducing end was not

Table 1 Chemical shifts of acetylated Xylp

\begin{tabular}{lllllll}
\hline Structural unit & $\mathbf{H 2}$ & $\mathbf{H 3}$ & $\mathrm{COCH}_{\mathbf{3}}$ & $\mathbf{C 2}$ & $\mathbf{C 3}$ & $\mathbf{C O C H}_{\mathbf{3}}$ \\
\hline -Xyl2-OAC- & 4.68 & - & 2.17 & 73.39 & - & 173.42 \\
-Xyl3-OAC- & - & 4.98 & 2.15 & - & 75.18 & 173.92 \\
-Xyl2,3-OAC- & 4.81 & 5.15 & $2.10 / 2.11$ & 71.30 & 72.78 & $173.04 / 173.53$ \\
-Xyl3-OAC2-MeGlcA- & - & 5.06 & 2.22 & - & 73.77 & 173.99 \\
\hline
\end{tabular}

Assignment of relevant chemical shifts of acetyl residues present in 4-O-methyl glucuronic acid (MeGlcA)-substituted xylo-oligosaccharides (AcUXOS) from E. globulus. made. On the ${ }^{1} \mathrm{H}$ NMR spectra, the signals corresponding to the acetyl groups present at the reducing and non-reducing end and on "internal" Xylp overlap. The ${ }^{1} \mathrm{H}$ NMR spectra obtained (Figure 4) showed separated resonances $(2.10 / 2.11,2.15,2.17$, and $2.22 \mathrm{ppm})$ for the different, acetylated structural units (-Xyl 2,3-O Ac-, -Xyl 3-O Ac-, -Xyl 2-O Ac-, and -Xyl 3-O Ac 2-MeGlcA-) present in AcUXOS (Table 1). By monitoring the changes in signal intensities for the region 2.1 to $2.22 \mathrm{ppm}$ during incubation with acetyl esterases, the deacetylation efficiency and preference towards differently acetylated Xyl $p$ can be investigated.

\section{Positional specificity}

The distribution of acetyl moieties over 2-O, 3-O, and 2,3-di-O acetylated $\mathrm{Xyl} p$ and 3-O acetylated Xyl $p$ 2-O substituted with MeGlcA and the free acetic acid obtained after an incubation of $16 \mathrm{~h}$ (endpoint) of AcUXOS with acetyl esterases from different families is shown in Figure 5. This distribution was obtained by monitoring the changes in signal intensities for the region 2.1 to $2.22 \mathrm{ppm}$, corresponding to the structural units (Figure 4). The acetyl esterases can be divided in three groups with respect to their positional specificity. The three groups showed activity towards 2-O and 3-O acetylated $\mathrm{Xyl} p$ or towards 2-O, 3-O, and 2,3-di-O acetylated Xyl $p$ or towards 2-O,3-O, and 2,3-di-O acetylated $\mathrm{Xyl} p$ and 3-O acetylated $\mathrm{Xyl} p \quad 2-O$ substituted with MeGlcA. The deacetylation of 2,3-di-O acetylated Xyl $p$ generates 2-O and 3-O acetylated Xyl $p$ moieties. Even though doubly acetylated $\mathrm{Xyl} p$ represents a relatively small fraction of the total acetylation (15\%), the deacetylation efficiency determined must be interpreted in the context of small amounts of 2-O and 3-O acetylated $\mathrm{Xyl} p$ being generated if the enzyme is active on diacetylated Xyl $p$. In the case of $T e C E 1, T r C E 5$, and $A n C E 5,2-$ $O$, 3-O, and 2,3-di- $O$ acetylated $\mathrm{Xyl} p$ were nearly completely deacetylated, as discussed below. Therefore, the generation of 2-O and 3-O acetylated $\mathrm{Xyl} p$ from 2,3-di$O$ acetylated $\mathrm{Xyl} p$ is not relevant regarding the deacetylation efficiencies obtained for these enzymes. However, for OsCE6 and AnCE16, the deacetylation efficiency towards $2-O$ and 3-O acetylated $\mathrm{Xyl} p$ may be slightly underestimated, as small amounts of 2-O and 3-O acetylated $\mathrm{Xyl} p$ were generated. Monitoring of the initial $30 \mathrm{~min}$ of the hydrolysis, as discussed below, allowed a clear determination of the activity of esterases towards different acetylated Xyl $p$.

\section{Acetyl esterases group I: activity exclusively towards 2-O and 3-O acetylated Xylp}

The bacterial acetyl esterases from $C$. thermocellum (CtCE2, CtCE3, and CtCE4) showed deacetylation of 2$O$ and 3-O acetylated Xylp. No activity was observed 
a
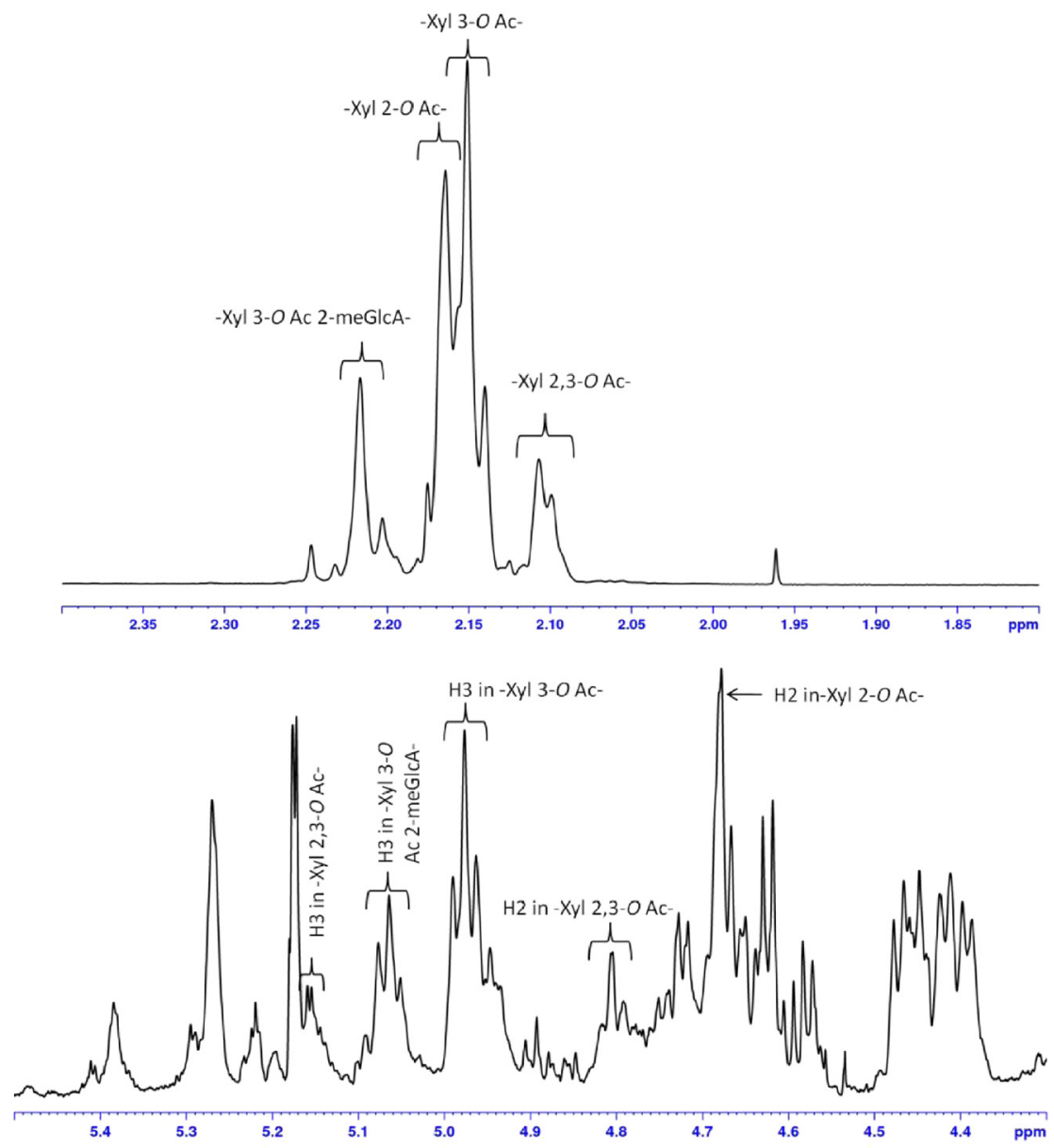

b

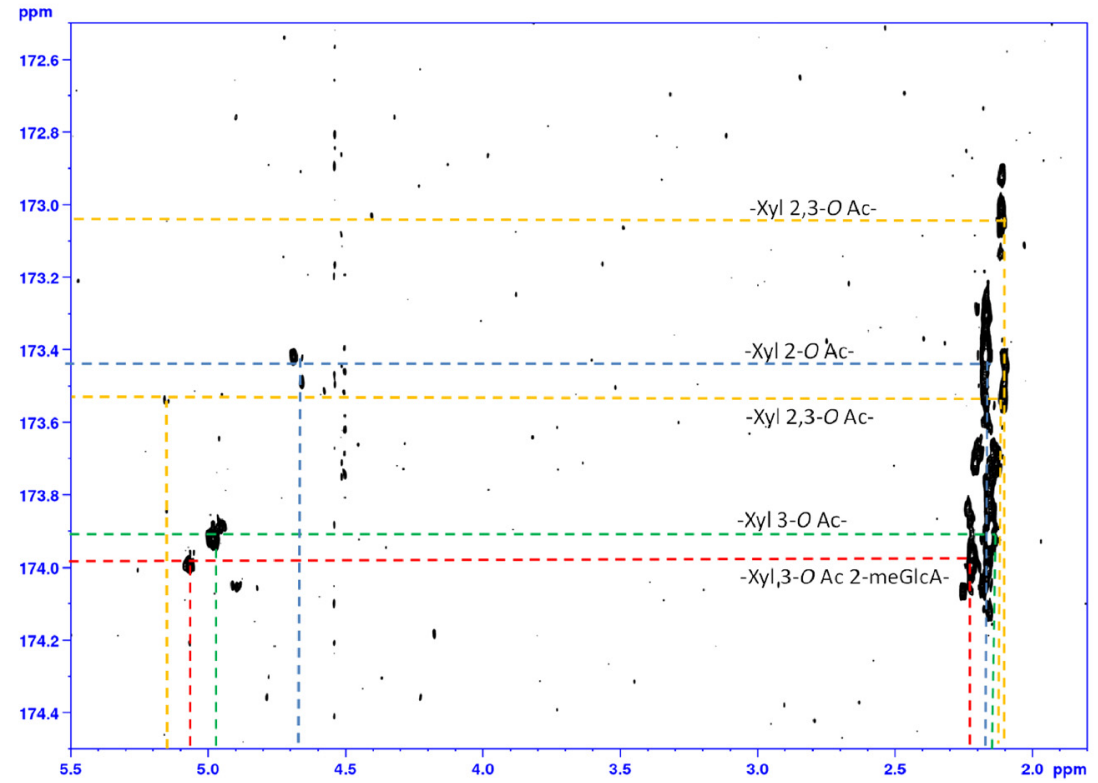

Figure 4 NMR spectra of acetylated 4-O-methyl glucuronic acid-substituted xylo-oligosaccharides. (a) ${ }^{1} \mathrm{H}$ NMR and (b) ${ }^{13} \mathrm{C} \mathrm{HMBC} \mathrm{spectra} \mathrm{of}$ acetylated 4-O-methyl glucuronic acid (MeGlcA)-substituted xylo-oligosaccharides (AcUXOS) from E. globulus. Designations are the same as in Table 1. 


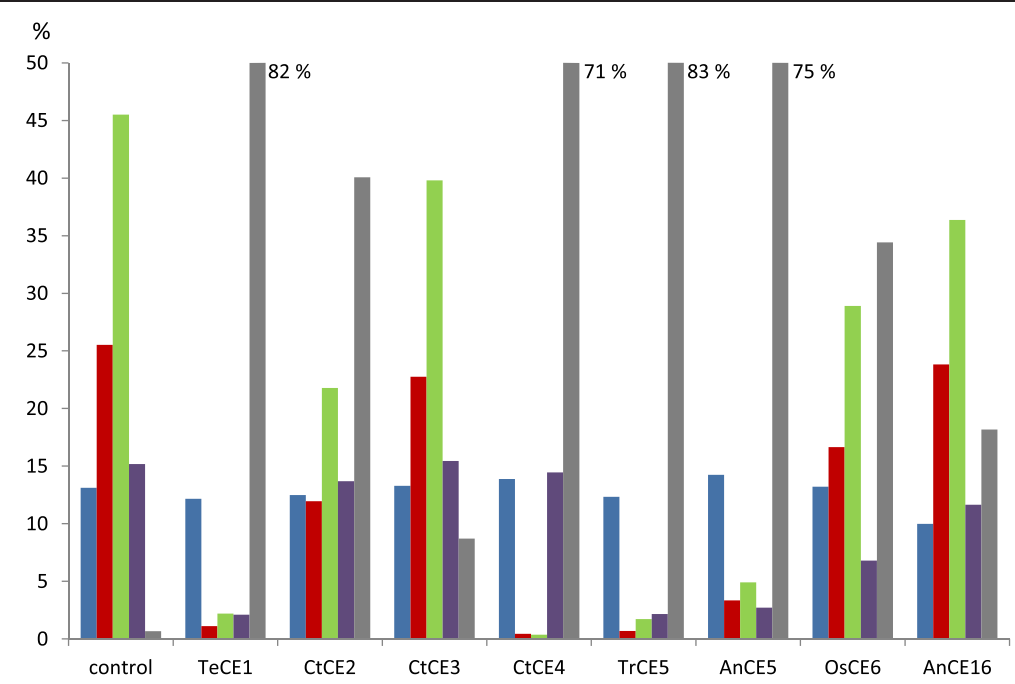

Figure 5 Deacetylation by acetyl esterases from different carbohydrate esterase families. Distribution of acetyl moieties over 2-O, 3-O, and 2,3-di-O acetylated Xylp, and 3-O acetylated Xylp a-1,2 substituted with MeGlcA present in acetylated 4-O-methyl glucuronic acid (MeGlcA)substituted xylo-oligosaccharides (AcUXOS) from E. globulus and free acetic acid as proportion (\%) of the sum of the total acetyl groups present after incubation of AcUXOS with acetyl esterases from different CE families (incubation time: 16 h). Structural units: (blue) -Xyl 3-O Ac 2-MeGlcA-, (red) -Xyl 2-O Ac-, (green) -Xyl 3-O Ac-, (purple) -Xyl 2,3-O Ac-, (gray) acetic acid; the control represents AcUXOS without enzyme treatment.

towards 2,3-di- $O$ acetylated $\mathrm{Xyl} p$ and 3-O acetylated Xyl $p$ 2-O substituted with MeGlcA. Complete deacetylation of $2-O$ and 3-O acetylated $\mathrm{Xyl} p$ was obtained for CtCE4. Hydrolysis of the acetyl groups at these positions has also been described for the CE4 acetyl esterase from S. lividans on aspen oligosaccharides [5,6]. The activity of the enzyme was shown to be metal ion-dependent and the enzyme was able to deacetylate positions 2 and 3 from the synthetic substrate methyl $\beta$-Dxylopyranoside $[20,21]$. As the ${ }^{1} \mathrm{H}$ signals show complete deacetylation of $2-O$ and 3-O acetylated $\mathrm{Xyl} p$, activity of $C t \mathrm{CE} 4$ towards terminal and internal acetylated xylopyranosyl units can be concluded. The presence of terminal acetylated xylo-oligosaccharides in AcUXOS has previously been shown by mass spectrometry $[8,10]$. Apparently, activity towards $2-O$ and 3-O acetylated Xyl $p$ was not hindered by substituents (such as MeGlcA) that could be present on neighboring xylopyranosyl units. A moderate deacetylation efficiency of these positions (52\% of both the 2-O and 3$O$ acetylated Xyl $p$, were deacetylated) was observed for $C t \mathrm{CE} 2$. CE2-classified esterases were described to act as 6-O-deacetylases, deacetylating the 6 position of hexopyranosyl residues [22]. Therefore, the activity towards acetylated xylan might only be a fraction of the activity towards other acetylated types of polysaccharides [3]. CtCE2 from $C$. thermocellum has a somewhat unique position within the CE2 family, as the active site acts as an esterase and cellulose binding domain, being an example of "gene sharing" [23]. Therefore, further studies on the deacetylation activity of other representatives of the CE2 family are required in order to determine whether this dual activity might affect the deacetylation efficiency and specificity compared to other members of this family. Activity on the synthetic substrates (4-O-acetyl 4-nitrophenyl $\beta$-Dxylopyranoside, methyl 3,4-O-diacetyl- and methyl $2,4-O$-diacetyl- $\beta$-D-xylopyranoside) has been reported for $C t C E 2$, suggesting a preference towards the 4-O position, which was also observed for the CE2 esterase from Cellvibrio japonicus [22]. However, in AcUXOS, the 4-O position is not acetylated. The data show that in the absence of the 4-O acetyl group, a clear decrease of the 2-O and 3-O acetylated Xylp, representing approximately $40 \%$ of the total acetylation present in AcUXOS, was obtained with CtCE2. Figure 3 shows that $C t C E 3$ was also able to hydrolyze both $2-O$ and 3-O acetylated Xyl $p$, although the deacetylation efficiency was low under the conditions applied. Approximately $9 \%$ of the total acetylation present in AcUXOS was removed. The activity by $C t C E 3$ towards acetylated xylo-oligosaccharides has previously been shown by its activity towards birchwood xylan [24].

\section{Acetyl esterases group II: activity towards 2-0, 3-0, and 2,3-di-O acetylated Xylp}

Clearly decreased ${ }^{1} \mathrm{H}$ signals for 2-O, 3-O, and 2,3-di-O acetylated Xylp were obtained after incubation of AcUXOS with TeCE1, $\operatorname{TrCE} 5, A n C E 5$, and OsCE6, showing deacetylation of $\mathrm{Xyl} p$ at these positions. In contrast, no activity towards the 3-O acetylated Xyl $p 2$ $O$ substituted with MeGlcA was observed. 
For $T e C E 1, T r C E 5$, and $A n C E 5$ the deacetylation at the 2-O, 3-O, and 2,3-di-O positions was nearly complete. As described above, complete deacetylation implies activity towards terminal and internal acetylated xylopyranosyl and also tolerance towards MeGlcA substituents that might be present on neighboring xylopyranosyl units. These three enzymes are efficiently deacetylating AcUXOS, releasing up to 83\% of the acetyl groups present in AcUXOS, with only the 3-O acetylated $\mathrm{Xyl} p$ 2-O substituted with MeGlcA (which comprises $13 \%$ of the total acetyl residues initially present) being recalcitrant towards hydrolysis.

\section{Acetyl esterases group III: activity towards 2-0, 3-0, and 2,3-di-O acetylated Xylp and 3-O acetylated Xylp 2-O substituted with MeGIcA}

For $A n C E 16$, moderate deacetylation of AcUXOS was observed. However, the positional tolerance of this enzyme was the highest of the enzymes tested, as the signals corresponding to 2-O, 3-O, and 2,3-di- $O$ acetylated $\mathrm{Xyl} p$ and 3-O acetylated $\mathrm{Xyl} p \quad 2-O$ substituted with MeGlcA decreased during the hydrolysis. The decrease found for 2- $O$ acetylated $\mathrm{Xyl} p$ after an incubation time of $16 \mathrm{~h}$ was low (13\%), indicating that $A n \mathrm{CE} 16$ does not hydrolyze acetyl groups efficiently at this position. A low deacetylation activity of 2-O acetylated Xyl $p$ by the CE16 acetyl esterase from $T$. reesei was also observed on an artificial substrate [9]. Significantly, our data not only show a clear signal decrease for 3-O and 2,3-di-O acetylated $\mathrm{Xyl} p$, but also for the 3-O acetylated $\mathrm{Xyl} p 2-O$ substituted with MeGlcA. This is relevant, as previous studies using artificial substrates for the characterization of CE16 acetyl esterases did not include acetylated Xyl $p$ carrying a MeGlcA substituent on the same residue [9]. If the deacetylation by $A n C E 16$ proceeds primarily at the non-reducing end, this would explain the moderate deacetylation efficiency towards acetylated $\mathrm{Xyl} p$. This strengthens the hypothesis of a predominant exo-activity by CE16 acetyl esterases from $A$. niger (this study) and T. reesei [17]. The NMR data showed no clear positional specificity towards the acetylated positions, while no obvious decrease was observed for the signal corresponding to 2-O acetylated $\mathrm{Xyl} p$.

\section{Deacetylation rates for different acetylated xylopyranosyl units}

Endpoint values for the deacetylation of AcUXOS indicate the ability of the acetyl esterases to catalyze the hydrolysis of acetyl groups at a specific location, but do not reflect the positional preferences at the start of hydrolysis. In order to determine the positional preferences on xylo-oligosaccharides, the deacetylation rate of acetyl esterases from different $\mathrm{CE}$ families was measured for 2$O, 3-O$, and 2,3-di- $O$ acetylated $\mathrm{Xyl} p$ and 3-O acetylated
Xyl $p$ 2-O substituted with MeGlcA after short incubations (5 or $30 \mathrm{~min}$; Table 2). The effect of small amounts of 2-O and 3-O acetylated $\mathrm{Xyl} p$ being generated by acetyl esterases with activity towards 2,3-di- $O$ acetylated $\mathrm{Xyl} p$ was discussed above. However, this does not affect the initial deacetylation rates, as all acetylated $\mathrm{Xyl} p$ are present in excess at the start. Figure 6 shows an example of the hydrolysis curves obtained for the various acetyl positions by incubation with the CE5 acetyl xylan esterase $(A n C E 5)$ from $A$. niger for $30 \mathrm{~min}$. From these hydrolysis curves, the deacetylation rates for each acetylated position (acetyl groups $\mathrm{removed} / \mathrm{min} / \mathrm{mg}$ enzyme, at $\mathrm{pH} 5.0$ and $50^{\circ} \mathrm{C}$ ) were calculated based on the decrease of the resonance corresponding to 2-O, 3-O, and 2,3-di-O acetylated $\mathrm{Xyl} p$ and 3-O acetylated $\mathrm{Xyl} p$ 2-O substituted with MeGlcA. Enzyme-recalcitrant acetyl groups were identified by the presence of a constant signal over the time period, as observed for 3-O acetylated $\mathrm{Xyl} p$ 2-O substituted with MeGlcA, in the hydrolysis curve of the sample incubated with AnCE5 (Figure 6). An additional file shows the hydrolysis curves obtained by incubation with acetyl esterases from different $\mathrm{CE}$ families in more detail (see Additional file 1).

Table 2 shows the rates of hydrolysis for the positions investigated. The values obtained (single point measurements) are a guide to their true specific activity. High deacetylation rates were observed for 2-O and 3-O acetylated $\mathrm{Xyl} p$ residues by $A n \mathrm{CE} 5, \operatorname{TrCE} 5$, and $C t C E 4$. Moreover, $A n C E 5$ and $\operatorname{TrCE} 5$ also showed high rates for the hydrolysis of the diacetylated xylopyranosyl units. Lower deacetylation rates were observed for TeCE1 and OsCE6 compared to $A n \mathrm{CE} 5$ or $\operatorname{TrCE} 5$. Finally, low rates were obtained for the bacterial CtCE2 and CtCE3, and these enzymes were not active on diacetylated Xyl $p$. In the case of $\mathrm{CtCE} 3$, activity on 2-O and 3-O acetylated $\mathrm{Xyl} p$ could only be concluded from the overnight incubation (Figure 5).

Regarding $A n C E 16$, a relatively low activity towards 2$O$, 3-O, and 2,3-di-O acetylated Xyl $p$ and 3-O acetylated $\mathrm{Xyl} p$ 2-O substituted with MeGlcA was observed from monitoring the first $30 \mathrm{~min}$ of the hydrolysis (Table 2). Also in this case, hydrolysis at these positions was clearly observed with the overnight incubation (Figure 5).

\section{Positional preference on acetylated xylo-oligosaccharides}

In order to evaluate whether acetyl esterases showed a preference for certain positions, the ratio of the deacetylation rate and the relative abundance of the acetyl groups present at a specific position was calculated (Table 3). For some enzymes (CtCE2, CtCE3, and $A n C E 16)$, the deacetylation activity was too low to determine clear positional preferences.

The data in Table 3 indicate a clear preference towards doubly acetylated Xyl $p$ for TeCE1. A preference towards 
Table 2 Deacetylation rates and rates for acetic acid released

\begin{tabular}{|c|c|c|c|c|c|}
\hline enzyme & $\begin{array}{l}\text {-Xyl3-OAc2- } \\
\text { MeGIcA- }\end{array}$ & $\begin{array}{l}-X y l 2- \\
\text { OAc- }\end{array}$ & $\begin{array}{l}\text {-Xyl3- } \\
\text { OAc- }\end{array}$ & $\begin{array}{l}\text {-Xyl2,3- } \\
\text { OAc- }\end{array}$ & $\begin{array}{l}\text { Acetic } \\
\text { acid }\end{array}$ \\
\hline$\overline{\mathrm{TeCE} 1^{\mathrm{a}}}$ & 0.0 & 12.7 & 25.5 & 44.0 & 74.6 \\
\hline $\mathrm{CtCE} 2^{\mathrm{b}}$ & 0.0 & $<0.01$ & 6.5 & 0.0 & 5.7 \\
\hline $\mathrm{CtCE}^{\mathrm{b}}$ & 0.0 & $<0.01$ & $<0.01$ & 0.0 & $<0.01$ \\
\hline $\mathrm{CtCE} 4^{\mathrm{b}}$ & 0.0 & 88.1 & 356.6 & 0.0 & 365.7 \\
\hline$A n C E 5^{a}$ & 0.0 & 202.7 & 276.2 & 274.4 & 754.7 \\
\hline $\operatorname{TrCE} 5^{\mathrm{a}}$ & 0.0 & 171.8 & 289.2 & 92.5 & 540.9 \\
\hline OsCE6 ${ }^{a}$ & 0.0 & 48.64 & 92.2 & 50.3 & 117.7 \\
\hline$A n C E 16^{b}$ & 0.03 & 1.80 & 1.92 & 1.50 & 5.25 \\
\hline
\end{tabular}

Deacetylation rates ( $\mu \mathrm{mol}$ acetyl groups removed $/ \mathrm{min} / \mathrm{mg}$ enzyme) by acetyl esterases for 2-O, 3-O, and 2,3-di-O acetylated Xylp and 3-O acetylated Xylp 2-O substituted with MeGlcA from eucalyptus and rates for the total acetic acid released ( $\mu \mathrm{mol}$ acetic acid released $/ \mathrm{min} / \mathrm{mg}$ enzyme).

${ }^{a} \mu \mathrm{mol}$ acetyl groups removed $/ \mathrm{min} / \mathrm{mg}$ enzyme and acetic acid released $/ \mathrm{min} /$ mg enzyme after $5 \mathrm{~min}$.

${ }^{b} \mu \mathrm{mol}$ acetyl groups removed/min/mg enzyme and acetic acid released $/ \mathrm{min} /$ $\mathrm{mg}$ enzyme after $30 \mathrm{~min}$.

diacetylated xylopyranosyl units has also been described for the CE1 acetyl xylan esterase from S. commune [6]. For $C t C E 4$, a preference for the 3-O acetylated $\mathrm{Xyl} p$ was observed, with a ratio more than twice as high as that for the 2-O acetylated Xylp. AnCE5 and OsCE6 showed a preference for 2,3-di-O acetylated $\mathrm{Xyl} p$ over 2-O and 3-O acetylated Xyl $p$. No such positional preference was observed for $\operatorname{TrCE} 5$, which deacetylated all positions with similar preference (except 3-O acetylated Xylp 2-O substituted with MeGlcA). The difference in preference of the CE5 esterases $\operatorname{Tr} \mathrm{CE} 5$ and $A n \mathrm{CE} 5$ shows that acetyl xylan esterases belonging to the same CE family but different species do not necessarily have the same positional preference. Consequently, sequence-based classification of esterases cannot fully predict the positional preferences of

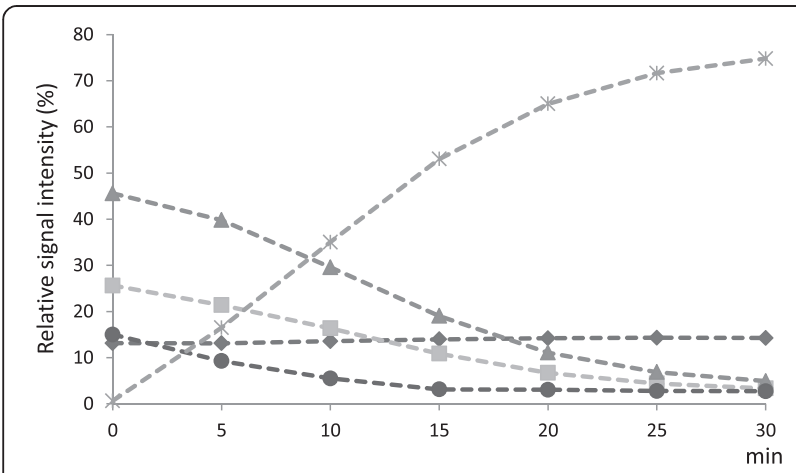

Figure 6 Deacetylation by acetyl xylan esterase from carbohydrate esterase family $\mathbf{5}$. Relative signal intensities (\% area of the total area of acetyl groups and acetic acid present) for acetyl xylan esterase CE5 (A. niger)-treated acetylated xylo-oligosaccharides. 3-O (triangles), 2-O (squares), 2,3-di-O (circles) acetylated Xylp, 3-O acetylated Xylp 2-O substituted with MeGlcA (diamonds), and acetic acid released (asterisks) these enzymes. Multiple representatives of one enzyme class will need to be compared in order to conclude whether positional preferences are conserved for different CE families.

The aim of this study was to determine the specificity (positional preference) of a number of acetyl esterases from different $\mathrm{CE}$ families. As the main indicator for this we used the rates of hydrolysis shown in Table 2. To allow the use of this information in industrial practice, incubation conditions were used that may be considered relevant for biofuel production $[12,13]$. These conditions do not necessarily reflect the $\mathrm{pH}$ and temperature optima of a particular esterase. It is clear that this would affect the absolute activity measured. It is less clear whether this could also affect the positional preference, and this limitation must be considered when using the preferences reported here.

\section{Conclusions}

Depending on the acetylation pattern of a particular substrate, suitable acetyl esterases may be selected from these data (Tables 2 and 3). Knowledge of the deacetylation efficiency and the positional preference of fungal and bacterial acetyl esterases allows the design of optimized enzyme mixtures. The acetyl esterases tested could be categorized into three groups with activities towards (i) 2-O and 3-O and (ii) 2-O, 3-O, and 2,3-di-O acetylated $\mathrm{Xyl} p$, and (iii) 2-O, 3-O, and 2,3-di-O acetylated Xyl $p$ and 3-O acetylated Xyl $p$ 2-O substituted with MeGlcA. High deacetylation rates were obtained for CtCE4, $\operatorname{TrCE5}$, and AnCE5 (Table 2) under the conditions employed. Positional preferences towards 2,3-di-O acetylated Xyl $p$ (TeCE1, $A n C E 5$, and OsCE6) or 3-O acetylated Xylp (CtCE4) were found. For the deacetylation of acetylated 4-O-methyl glucuronic acid-substituted xylooligosaccharides a combination of the CE5 (TrCE5 or

Table 3 Positional preferences of acetyl xylan esterases

\begin{tabular}{lllll}
\hline Enzyme & $\begin{array}{l}-\mathbf{X y l 3 - O A c 2 -} \\
\text { MeGlcA- }\end{array}$ & $\begin{array}{l}-\mathbf{X y l 2}- \\
\text { OAc- }\end{array}$ & $\begin{array}{l}-\mathbf{X y l 3 -} \\
\text { OAc- }\end{array}$ & $\begin{array}{l}-\mathbf{X y l 2 , 3 -} \\
\text { OAc- }\end{array}$ \\
\hline TeCE1 & 0 & 12 & 14 & 74 \\
CtCE2 & 0 & n.d. & n.d. & 0 \\
CtCE3 & 0 & n.d. & n.d. & 0 \\
CtCE4 & 0 & 30 & 70 & 0 \\
AnCE5 & 0 & 24 & 19 & 57 \\
TrCE5 & 0 & 35 & 33 & 32 \\
OsCE6 & 0 & 26 & 28 & 46 \\
AnCE16 & n.d. & n.d. & n.d. & n.d. \\
\hline
\end{tabular}

Positional preference on acetylated xylo-oligosaccharides, calculated as the positional rate per relative amount of acetyl groups present (2-O, 3-O, 2,3-di-O acetylated Xylp and 3-O acetylated Xylp 2-O substituted with MeGlcA) at the start of hydrolysis. The data were normalized.

0 , no activity; n.d., not determined. 
$A n \mathrm{CE} 5)$ and the CE16 acetyl esterases (AnCE16) would seem to be the most efficient.

\section{Methods}

\section{Substrates}

Eucalyptus globulus xylan hydrolysate was kindly donated by Dr. J.C. Parajo of the University of Vigo-Ourense, Spain [25]. The acetylated 4-O-methyl glucuronic acid (MeGlcA)-substituted xylo-oligosaccharide (AcUXOS) fraction of the xylan hydrolysate was described previously [8]. The Xyl, Ara, Gal, and MeGlcA contents of AcUXOS were 765, 0, 42, and $207 \mathrm{mg} \mathrm{g}^{-1}$ dry matter (DM) [8]. The acetic acid was measured by ${ }^{1} \mathrm{H}$ NMR, and was $210 \mathrm{mg} \mathrm{g}^{-1} \mathrm{DM}$. Corn silage water unextractable solids (WUS) were used and have been described [1].

\section{Enzymes}

Cloning and expression of the acetyl esterase 1 from Trichoderma reesei belonging to CE family 5 ( $\operatorname{Tr} \mathrm{CE} 5, T$. reesei, CAA93247.1) has been described previously [15]. Acetyl esterases from Aspergillus niger belonging to $\mathrm{CE}$ families 5 and 16 [AnCE5 (CAK49022.1), AnCE16 (CAK45102.1)] and the CE1 classified acetyl esterase from Talaromyces emersonii [TeCE1 (ADX07526.1)] were obtained from DSM (Heerlen, The Netherlands). Expression of these genes in A. niger ISO527 was performed according to the procedure described previously [15]. Acetyl esterases classified as belonging to $\mathrm{CE}$ families 2 [CtCE2 (AAA23224)], 3 [CtCE3 (ABN52033)], and 4 [CtCE4 (ABN54169)] from Clostridium thermocellum were purchased from Prozomix (Haltwhistle, UK). The CE6 classified acetyl esterase [OsCE6 (AAC14690.1)] from Orpinomyces sp. PC-2 was purchased from Megazyme (Wicklow, Ireland).

\section{Electrophoresis}

SDS-PAGE was performed with a NuPAGE 10\% Bis-Tris gel (Life Technologies, Carlsbad, CA, USA) and a Power Ease 500 system (Life Technologies). The protein bands were stained with the Instant Blue protein gel stain (Expedeon Inc., San Diego, CA, USA). The protein markers Mark 12 and SeeBlue Plus 2 (Life Technologies) were used. Quantification of the protein bands obtained by densitometry was performed with the software ImageJ, developed at the National Institute of Health (Bethesda, MD, USA).

\section{Calculation of molecular masses of proteins}

The protein sequences of the acetyl esterases [TeCE1, $A n C E 5, T r C E 5$, and $A n C E 16]$ were retrieved from the National Center for Biotechnology Information (Bethesda, MD, USA, www.ncbi.nlm.nih.gov) and calculated with the Compute pI/Mw tool (ExPASy Bioinformatics Resource Portal, www.expasy.org).

\section{$p$-Nitrophenyl acetate ( $p$-NP-Ac) assay}

Enzyme assays with the acetyl esterases using $p$-NP-Ac were performed as described previously [15]. The enzymes were applied at a dosage of $0.3 \mathrm{mg}$ total protein $\mathrm{mL}^{-1}$ in sodium acetate buffer $(10 \mathrm{mM}, \mathrm{pH} 5.0)$ at $40^{\circ} \mathrm{C}$. The $p$-nitrophenol release was determined by continuous measurement for $10 \mathrm{~min}$ at $405 \mathrm{~nm}$.

\section{Monosaccharide-releasing activities assay}

WUS $\left(10 \mathrm{~g} \mathrm{~L}^{-1}\right)$ was incubated with acetyl xylan esterases (10 g protein $\mathrm{kg}^{-1}$ substrate) in sodium citrate buffer (10 mM, pH 5.0, containing sodium azide $0.5 \mathrm{~g} \mathrm{~kg}^{-1}$ ) at $50^{\circ} \mathrm{C}$ and $700 \mathrm{rpm}$ for $8 \mathrm{~h}$. The enzymes were heat inactivated $\left(10 \mathrm{~min}, 98^{\circ} \mathrm{C}\right)$ and analyzed for the presence of monosaccharides ( $\mathrm{mg}$ monosaccharides released $\mathrm{g}^{-1}$ dry matter) on an HPAEC system as described previously [1].

\section{NMR}

${ }^{1} \mathrm{H}$ and $2 D$ NMR: ${ }^{1} \mathrm{H}_{-}{ }^{13} \mathrm{C}$ HSQC spectra were recorded on an Avance III $700 \mathrm{MHz}$ spectrometer (Bruker BioSpin, Billerica, MA, USA), equipped with a helium-cooled cryoprobe. The HSQC spectra were recorded using a correlation via double inept transfer pulse program using sensitivity improvement (hsqcetgpsi2), in 32 scans, with 32 dummy scans, 512 increments, sweep width 130 ppm, relaxation delay $1.2 \mathrm{~s}$, and acquisition time $0.36 \mathrm{~s}$.

${ }^{1} \mathrm{H}_{-}{ }^{13} \mathrm{C}$ HMBC spectra were recorded using heteronuclear zero and double quantum coherence with a twofold low-pass J-filter constant time version (shmbcctetgpl2nd), in 64 scans, with 16 dummy scans, 1024 increments, sweep width $20 \mathrm{ppm}$, relaxation delay $1.2 \mathrm{~s}$, and acquisition time $0.24 \mathrm{~s}$.

\section{NMR enzyme assay}

AcUXOS was dissolved in deuterated sodium citrate buffer $(10 \mathrm{mM}, \mathrm{pH} 5.0)$ at a concentration of $5 \mathrm{~g} \mathrm{~L}^{-1}$, transferred to NMR tubes ( $3 \times 103.5 \mathrm{~mm}$ glass tubes) and heated to $50^{\circ} \mathrm{C}$. A ${ }^{1} \mathrm{H}$ NMR spectrum of each sample was measured before the addition of enzymes. A concentration of $5 \mathrm{~g} \mathrm{~kg}^{-1}$ substrate for the acetyl esterases TeCE1, CtCE2, CtCE3, CtCE4, TrCE5, AnCE5, OsCE6, and $A n C E 16$ was determined as suitable for the purpose of measurement. CtCE4 was also dosed at $0.1 \mathrm{~g} \mathrm{~kg}^{-1}$ substrate, as this enzyme showed a high hydrolytic rate (data not shown). The samples were vortex mixed after addition of the acetyl esterases. The incubations were done at $50^{\circ} \mathrm{C}$ and monitored by continuous measurement of the ${ }^{1} \mathrm{H}$ NMR spectra for $30 \mathrm{~min}$ and a single measurement after $16 \mathrm{~h}$ on an Avance III $700 \mathrm{MHz}$ spectrometer (Bruker).

\section{Calculation of deacetylation rates}

The concentration of acetic acid present in the hydrolysate was calculated from the ${ }^{1} \mathrm{H}$ NMR spectrum as 
described previously [26] using the integral area of the internal standard 4,4-dimethyl-4-silapentane-1-sulfonic acid. Concentrations of 2-O, 3-O, and 2,3-di- $O$ acetylated Xyl $p$ residues and 3-O acetylated Xyl $p$ residues 2$O$ substituted with MeGlcA were calculated accordingly based on the resonances obtained by ${ }^{1} \mathrm{H}$ NMR analysis; the values were $2.17,2.15,2.11 / 2.10$, and $2.22 \mathrm{ppm}$, respectively. The rates of the acetic acid release and the deacetylation rates for the different locations of acetyl within the $\mathrm{Xyl} p$ residues (2-O, 3-O, 2,3-di-O acetylated $\mathrm{Xyl} p$, and the 3-O acetylated $\mathrm{Xyl} p$ 2-O substituted with MeGlcA) were calculated as $\mu \mathrm{mol}$ acetic acid released/ $\mathrm{min} / \mathrm{mg}$ enzyme and $\mu \mathrm{mol}$ specific acetyl group re$\mathrm{moved} / \mathrm{min} / \mathrm{mg}$ enzyme, respectively, after a measurement time of $5 \mathrm{~min}$ for the relatively fast acetyl esterases (TeCE1, TrCE5, AnCE5, and OsCE6), dosed at 5 g protein $\mathrm{kg}^{-1}$ substrate, and after $30 \mathrm{~min}$ for the relatively slow acetyl esterases (CtCE2, CtCE3, and AnCE16), dosed at $5 \mathrm{~g}$ protein $\mathrm{kg}^{-1}$ substrate, and for the CtCE4, dosed at $0.1 \mathrm{~g}$ protein $\mathrm{kg}^{-1}$ substrate. The purity of acetyl esterases present in the enzyme solutions was determined by quantitative densitometry of the gels obtained by the SDS-PAGE analysis, as described above. The hydrolytic rates were calculated per actual mg acetyl esterase present.

\section{Additional file}

Additional file 1: Positional preferences of acetyl esterases from different CE families towards 4-O-methyl glucuronic acidsubstituted xylo-oligosaccharides. Relative signal intensities (\% area of the total area of acetyl groups and acetic acid present) for enzyme treated acetylated xylo-oligosaccharides with acetyl esterase from different CE families. 3-O (triangles), 2-O (squares), 2,3-di-O (circles), acetylated Xylp, 3-O acetylated Xylp 2-O substituted with MeGlcA (diamonds), and acetic acid released (asterisks).

\section{Abbreviations \\ AcUXOS: acetylated 4-O-methyl glucuronic acid-substituted xylo- oligosaccharides; AnCE5: CE5 classified acetyl esterase from Aspergillus niger; AnCE16: CE16 classified acetyl esterase from Aspergillus niger; CtCE2: CE2 classified acetyl esterase from Clostridium thermocellum; CtCE3: CE3 classified acetyl esterase from Clostridium thermocellum; CtCE4: CE4 classified acetyl esterase from Clostridium thermocellum; DM: dry matter; HMBC spectroscopy: heteronuclear multiple-bond correlation spectroscopy; HPAEC: high-performance anion exchange chromatography; HSQC spectroscopy: heteronuclear single quantum coherence spectroscopy; MeGlcA: methyl glucuronic acid; NMR: nuclear magnetic resonance; OsCE6: CE6 classified acetyl esterase from Orpinomyces sp. PC-2; $p$-NP-Ac: p-nitrophenyl acetate; SDS-PAGE: sodium dodecyl sulfate polyacrylamide gel electrophoresis; TeCE1: CE1 classified acetyl esterase from Talaromyces emersonii; TrCE5: CE5 classified acetyl esterase from Trichoderma reesei; WUS: water unextractable solids; Xylp: xylopyranosyl unit.}

\section{Competing interests}

The authors declare that they have no competing interests.

\section{Authors' contributions}

KGN, HAS, and HS designed and coordinated the study. KGN, JHJR, and ACS carried out the experiments and data analysis. KGN prepared the manuscript.
HAS, HS, ACS, and HG contributed to the manuscript preparation. All authors read and approved the final version of the manuscript.

\section{Acknowledgements}

The research leading to these results has received funding from the (European Community's) Seventh Framework Programme (FP7/2007-2013) under grant agreement 238084.

\section{Author details}

${ }^{1}$ DSM Biotechnology Center, PO Box 12600 MA Delft, The Netherlands. ${ }^{2}$ Laboratory of Food Chemistry, Wageningen University, Bornse Weilanden 9, 6708 WG Wageningen, The Netherlands.

Received: 23 September 2014 Accepted: 15 December 2014 Published online: 22 January 2015

\section{References}

1. Neumüller KG, Streekstra H, Schols HA, Gruppen H. Synergistic action of enzyme preparations towards recalcitrant corn silage polysaccharides. Biomass Bioenerg. 2014;60:88-97.

2. Lombard V, Golaconda Ramulu H, Drula E, Coutinho PM, Henrissat B. The carbohydrate-active enzymes database (CAZy) in 2013. Nucleic Acids Res. 2014:42:D490-495

3. Biely P. Microbial carbohydrate esterases deacetylating plant polysaccharides. Biotechnol Adv. 2012;30:1575-88.

4. Pawar PM, Koutaniemi S, Tenkanen M, Mellerowicz EJ. Acetylation of woody lignocellulose: significance and regulation. Front Plant Sci. 2013;4:118. doi:10.3389/fpls.2013.00118.

5. Uhliariková I, Vršanská M, McCleary BV, Biely P. Positional specificity of acetylxylan esterase on natural polysaccharide: an NMR study. Biochim Biophys Acta. 2013;1830:3365-72.

6. Pouvreau L, Jonathan MC, Kabel MA, Hinz SWA, Gruppen H, Schols HA. Characterization and mode of action of two acetyl xylan esterases from Chrysosporium lucknowense C1 active towards acetylated xylans. Enzyme Microb Tech. 2011;49:312-20.

7. Koutaniemi S, van Gool MP, Juvonen M, Jokela J, Hinz SW, Schols HA, et al. Distinct roles of carbohydrate esterase family CE16 acetyl esterases and polymer-acting acetyl xylan esterases in xylan deacetylation. J Biotechnol. 2013;168:684-92.

8. Biely P, Mastihubová M, Tenkanen M, Eyzaguirre J, Li X, Vršanská M. Action of xylan deacetylating enzymes on monoacetyl derivatives of 4-nitrophenyl glycosides of $\beta$-D-xylopyranose and $a$-L-arabinofuranose. J Biotechnol. 2011;151:137-42.

9. Neumüller KG, Streekstra H, Gruppen H, Schols HA. Trichoderma longibrachiatum acetyl xylan esterase 1 enhances hemicellulolytic preparations to degrade corn silage polysaccharides. Bioresource Technol. 2014;163:64-73.

10. Agbor VB, Cicek N, Sparling R, Berlin A, Levin DB. Biomass pretreatment: fundamentals toward application. Biotechnol Adv. 2011;29:675-85.

11. Sun RC. Cereal straw as a resource for sustainable biomaterials and biofuels. Amsterdam: Elsevier; 2010.

12. Taherzadeh MJ, Karimi K. Enzyme-based hydrolysis processes for ethanol from lignocellulosic materials: a review. Bioresources. 2007;2:707-38.

13. Sundberg M, Poutanen K. Purification and properties of two acetylxylan esterases of Trichoderma reesei. Biotechnol Appl Biochem. 1991;13:1-11.

14. Neumüller KG, Streekstra H, Gruppen H, Schols HA. Acetyl xylan esterase Axe1 (T. reesei, carbohydrate esterase family 5) supplemented to a (hemi) cellulolytic preparation enhances degradation of recalcitrant corn silage polysaccharides. Chem Eng Trans. 2014;37:277-82.

15. Darlymple BP, Cybinski DH, Layton I, McSweeney CS, Xue GP, Swadling YJ, et al. Three Neocallimastix patriciarum esterases associated with the degradation of complex polysaccharides are members of a new family of hydrolases. Microbiology. 1997;143:2605-14.

16. Biely P, Cziszárová M, Agger JW, Li XL, Puchart V, Vršanská M, et al. Trichoderma reesei CE16 acetyl esterase and its role in enzymatic degradation of acetylated hemicellulose. Biochim Biophys Acta. 2014;1840:516-25.

17. Cantarel BL, Lombard V, Henrissat B. Complex carbohydrate utilization by the healthy human microbiome. PLoS One. 2012;7:e28742.

18. Evtuguin DV, Tomás JL, Silva AM, Neto CP. Characterization of an acetylated heteroxylan from Eucalyptus globulus Labill. Carbohyd Res. 2003;338:597-604. 
19. Biely $P$, Mastihubová $M$, Puchart $V$. The vicinal hydroxyl group is prerequisite for metal activation of Clostridium thermocellum acetylxylan esterase. Biochim Biophys Acta. 2007;1770:565-70

20. Taylor EJ, Gloster TM, Turkenburg JP, Vincent F, Brzozowski AM, Dupont C, et al. Structure and activity of two metal ion-dependent acetylxylan esterases involved in plant cell wall degradation reveals a close similarity to peptidoglycan deacetylase. J Biol Chem. 2006;281:10968-75.

21. Montanier C, Money VA, Pires VMR, Flint JE, Pinheiro BA, Goyal A, et al. The active site of a carbohydrate esterase displays divergent catalytic and noncatalytic binding functions. PloS Biol. 2009; e1000071. doi:10.1371/ journal.pbio.1000071.

22. Topakas E, Kyriakopoulos S, Biely P, Hirsch J, Vafiadi C, Christakopoulos P. Carbohydrate esterases of family 2 are 6-O-deacetylases. FEBS Lett. 2010;584:543-8.

23. Correia MA, Prates JA, Brás J, Fontes CM, Newman JA, Lewis RJ, et al. Crystal structure of a cellulosomal family 3 carbohydrate esterase from Clostridium thermocellum provides insights into the mechanism of substrate recognition. J Mol Biol. 2008;379:64-72.

24. Biely P, Cziszárová M, Uhliariková I, Agger JW, Li XL, Eijsink VGH, et al. Mode of action of acetylxylan esterases on acetyl glucuronoxylan and acetylated oligosaccharides generated by a GH10 endoxylanase. Biochim Biophys Acta. 2013:1830:5075-86.

25. Gullón P, González-Muñoz MJ, Domínguez H, Parajó JC. Membrane processing of liquors from Eucalyptus globulus autohydrolysis. J Food Eng. 2008;87:257-65.

26. Neumüller KG, Carvalho de Souza A, Van Rijn J, Appeldoorn MM, Streekstra $\mathrm{H}$, Schols HA, et al. Fast and robust method to determine phenoyl and acetyl esters of polysaccharides by quantitative ${ }^{1} \mathrm{H}$ NMR. J Agric Food Chem. 2013;61:6282-7.

\section{Submit your next manuscript to BioMed Central and take full advantage of:}

- Convenient online submission

- Thorough peer review

- No space constraints or color figure charges

- Immediate publication on acceptance

- Inclusion in PubMed, CAS, Scopus and Google Scholar

- Research which is freely available for redistribution 